\title{
Association between Smoking and Overall and Specific Mortality in Patients with Bladder Cancer: A Population-based Study
}

\author{
Chung-Han Ho ${ }^{\mathrm{a}, \mathrm{b}, 1}$, Wen-Hsin Tseng ${ }^{\mathrm{c}, 1}$, Steven K. Huang ${ }^{\mathrm{c}}$, Chien-Liang Liu ${ }^{\mathrm{c}}$, Yu-Cih Wu ${ }^{\mathrm{a}}$, \\ Allen W. Chiu ${ }^{\text {d,e }}$ and Khaa Hoo Ong f,g,* \\ ${ }^{a}$ Department of Medical Research, Chi Mei Medical Center, Tainan, Taiwan \\ ${ }^{\mathrm{b}}$ Cancer Center, Wan Fang Hospital, Taipei Medical University, Taipei, Taiwan \\ ${ }^{\mathrm{c}}$ Division of Urology, Department of Surgery, Chi Mei Medical Center, Tainan, Taiwan \\ ${ }^{\mathrm{d}}$ Department of Urology, Mackay Memorial Hospital, Taipei, Taiwan \\ e School of Medicine, National Yang-Ming University, Taipei, Taiwan \\ ${ }^{\mathrm{f}}$ Division of General Surgery, Department of Surgery, Chi Mei Medical Center, Tainan, Taiwan \\ ${ }^{\mathrm{g}}$ Institute of Biomedical Science, National Sun Yat-Sen University, Kaohsiung, Taiwan
}

Received 13 July 2021

Accepted 19 January 2022

Pre-press 10 February 2022

Published 3 June 2022

\begin{abstract}
.
BACKGROUND: The role of smoking in the prognosis of bladder cancer may significantly impact clinical management. It is also a considerable burden to Taiwan's economy and health of its citizens.

OBJECTIVE: To search Taiwan's National Health Insurance Research Database to determine whether smoking affected overall and cancer-specific mortality of patients with bladder cancer.

METHODS: We collected data on basic information, tumor stage, and comorbidities. Each smoking case was propensity score-matched by age, sex, and diagnosis year to one control individual among bladder cancer patients. The study comprised a never-smoke and an ever-smoke group, with each group including 4,728 patients after matching. We evaluated the association between smoking and mortalities in patients with bladder cancer. Cox proportional regression modeling was used to estimate hazard ratios (HRs) of overall and cancer-specific mortality rates. Stratified analysis was also performed to estimate risk ratios of overall and cancer-specific mortalities in bladder cancer patients with and without a history of smoking history among different subgroups.

RESULTS: The overall and specific mortality ratio of patients who were ever smokers were 1.15-fold and 1.16-fold, respectively, compared with those of never smokers (overall: $95 \%$ confidence interval $[\mathrm{CI}], 1.06-1.26, P=0.0014$; specific: $95 \% \mathrm{CI}$, 1.03-1. 03, $P=0.0176)$. Patients with bladder cancer who smoked and had significantly higher overall and specific mortality rates were those with Charlson Comorbidity Index $(\mathrm{CCI}) \geq 3$ (overall: $P=0.0119$; specific: $P=0.0092$ ), diabetes mellitus (DM; overall: $P=0.0046$; specific: $P=0.0419)$, and non-muscle-invasive bladder cancer (NMIBC; overall: $P=0.0038$; specific: $P=0.0014)$.
\end{abstract}

\footnotetext{
${ }^{1}$ Co-first author: Chung-Han Ho, Wen-Hsin Tseng.

${ }^{*}$ Correspondence to: Khaa Hoo Ong, Division of General Surgery, Department of Surgery, Chi Mei Medical Center, Tainan, Taiwan; Institute of Biomedical Science, National Sun Yat-Sen
}

$\overline{\text { University, Kaohsiung, Taiwan, No. 901, Zhonghua Rd., Yongkang }}$ Dist., Tainan City 710, Taiwan (R.O.C.). Tel.: (+886)921594954; Fax: (+886)6 281 2811; E-mail: b101096108@tmu.edu.tw. 
CONCLUSIONS: Overall and specific mortality rates were significantly higher in the ever-smoke group than in the never-smoke group. The ever-smoke group with male sex, $\mathrm{CCI} \geq 3, \mathrm{DM}$, and NMIBC had increased risks for overall and specific mortality.

Keywords: Smoking, overall mortality, specific mortality, bladder cancer

\section{INTRODUCTION}

There are projected to be about 83,730 newly diagnosed bladder cancer patients in 2021 in the United States [1]. Bladder cancer was ranked sixth among all cancers in terms of incidence [1]. In addition, the number of bladder cancer-specific deaths was approximately 17,200, which ranked tenth among all types of cancer [1]. In Taiwan, bladder cancer is the ninth most common cancer, with approximately 1,600 new cases every year [2]. Cigarette smoking exposure is a major risk factor for bladder cancer; increasing the risk for bladder cancer by two- to fourfold [3, 4]. A recent study found that about $50 \%$ of young men and $10 \%$ of young women were smokers, and relatively few quit [5]. In Taiwan, although the government has implemented the 2009 Tobacco Hazards Prevention Act, tax increases, and warnings on tobacco packages, the smoking prevalence remains at $23.4 \%$ in men and $2.4 \%$ in women [2]. Thus, smoking is a considerable burden to Taiwan's health and economy.

Approximately $75 \%$ of patients with bladder cancer are with diagnosed with non-muscle-invasive bladder cancer (NMIBC) and are then treated with transurethral resection of bladder tumor (TURBT) followed by intravesical therapy [6]. In contrast, muscle-invasive bladder cancer (MIBC), which accounts for approximately $25 \%$ of newly diagnosed bladder cancers, is usually treated with radical cystectomy (RC) or trimodal therapy with maximal transurethral resection of the bladder tumor followed by concurrent chemoradiotherapy [6]. Continuous smoking is an independent predictor of disease recurrence in patients with either NMIBC and MIBC $[3,7$, 8]. Several studies have reported a high mortality rate in patients who underwent RC $[8,9]$. However, conclusive evidence of an association between smoking and mortality in NMIBC is lacking [7, 10].

Smoking can significantly impact the clinical management bladder cancer. Therefore, we used the Taiwan's National Health Insurance Research Database to conduct a propensity score-matched study with a long-term follow-up period. We compared the risk for overall and specific mortalities associated with bladder cancer patients who had a history of smoking with matched cohorts using this nationwide database.

\section{MATERIALS AND METHODS}

\section{Data source}

Datasets from the Taiwan Cancer Registry (TCR), the Taiwan's National Health Insurance Research Database (NHIRD), and the Taiwan's Cause-ofDeath Database were used in this study. These claims datasets were all from the Health and Welfare Data Science Center (HWDC), an integrated health-related database center. The TCR was established in 1979 to monitor Taiwan's cancer incidence and mortality rates. It includes information on individual demographics, cancer stages, cancer primary sites, tumor histology, and treatment types. NHIRD is based on Taiwan's national health insurance program, which includes detailed healthcare information of more than 99\% of Taiwan's population from 1996 to 2018. For research purposes, the HWDC released the deidentified claims to the public in an anonymous format. This study was conducted in compliance with the Declaration of Helsinki and has been approved by the Research Ethics Committee of Chi Mei Hospital (IRB no.11005-E01). In addition, patient informed consent was waived by the Research Ethics Committee of Chi Mei Hospital.

\section{Definitions of study subjects}

Patients with bladder cancer between 2000 and 2017 were selected from the TCR using the International Classification of Diseases for Oncology, 3rd edition (ICD-O-3): C67. Smoking information was included in the TCR from 2011; thus, patients who were diagnosed with bladder cancer before 2011 were excluded. In addition, patients with incomplete information in the TCR were excluded from this study. A flowchart summarizing the selection of study subjects is presented in Fig. 1. 


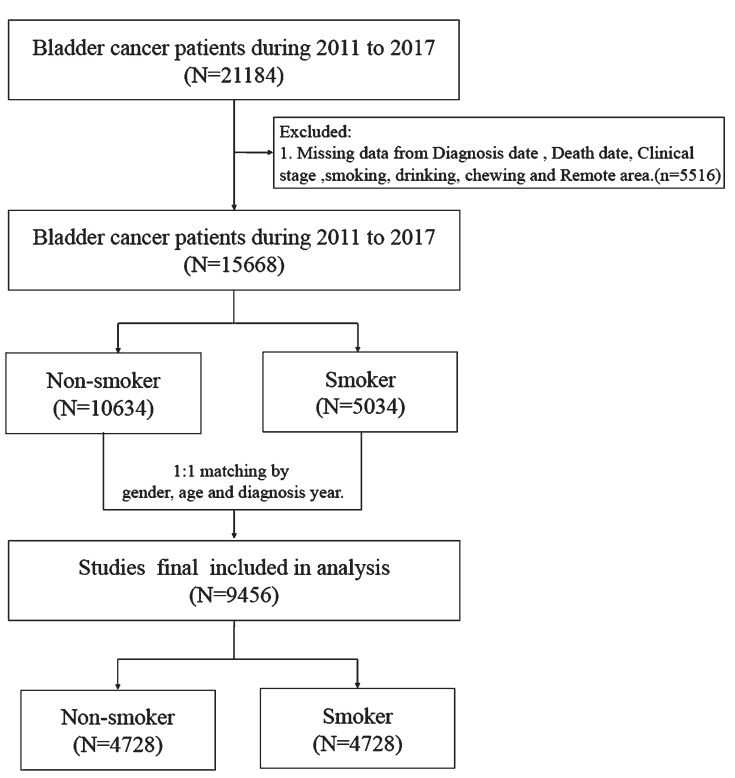

Fig. 1. Flow chart.

\section{Measurement}

The primary outcome of this study was mortality. Mortality was determined from the Cause-of-Death Dataset. The mortality risk was estimated using two endpoints: overall and cancer-specific mortalities. The time for the overall mortality was set from the date of bladder cancer diagnosis to the date of death, regardless of the cause. Cancer-specific mortality was defined as the cause of death due to bladder cancer. Considering the potential confounding effect of smoking, each smoking case was propensity scorematched by age, sex, and diagnosis year to one control individual among bladder cancer patients. The propensity score matching approach with a greedy algorithm was estimated using a multivariable logistic regression model to identify cases and controls [11, 12]. An SAS (SAS Institute, USA) matching macro, "\%OneToManyMTCH," was used for this caliper matching of nearest-neighbor approach for the first four to eight digits of propensity scores [13].

We followed up previous studies to find out the factors that are likely to affect bladder cancer development [14-17]. We separate the bladder cancer to NMIBC and muscle-invasive bladder cancer (MIBC). NMIBC was defined as patients with $\mathrm{T}$ stage 0 and 1 and MIBC was defined as patients with T stage 2, 3, 4 , and metastasis.

Patients with comorbidities were defined as patients with the diseases included below at least one year before the diagnosis of bladder cancer. This definition was based on the International Classification of Diseases, Ninth Revision, Clinical Modification (ICD-9-CM) codes in Appendix 1. Comorbidities included the following: myocardial infarction; congestive heart failure, peripheral vascular disease, cardiovascular disease, dementia, chronic obstructive pulmonary disease, renal disease, diabetes mellitus, hypertension, and liver disease.

\section{Statistical analysis}

Continuous variables are presented as means \pm standard deviations, while categorical variables are presented as frequencies with percentages. The distribution differences between bladder cancer with smoking and those without was compared using the Student's $t$-test for continuous variables and the Pearson's chi-square test for categorical variables. The trends of the overall and cancer-specific mortalities were plotted using the Kaplan-Meier method with the log-rank test to compare trend differences between patients with a history of smoking and those without. In addition, the Cox proportional regression model was used to estimate the hazard ratios (HRs) of the overall and cancer-specific mortalities. Adjusted HRs were calculated from the multivariable Cox regression with adjustment of age at diagnosis, sex, body mass index (BMI), Charlson Comorbidity Index (CCI) score, drinking, chewing betel nut, clinical stage, treatment type, and comorbidities. The stratified analysis was also presented to estimate the risk ratios of the overall and cancer-specific mortalities between bladder cancer patients with a smoking history and those without among different subgroups. To avoid violation of the proportional hazards assumption, the estimated HRs were checked using the Schoenfeld residuals test. The SAS 9.4 (SAS Institute, Inc, Cary, NC, USA) was used to perform all statistical analyses, while Stata software, version 12 (Stata Corp, College Station, TX, USA) was used to illustrate the Kaplan-Meier curves. A $P$-value $<0.05$ was set for statistical significance.

\section{RESULTS}

This study involved 9,456 patients who had bladder cancer after matching. The never-smoke and the ever-smoke groups each included 4,728 patients. The baseline characteristics of the never- and ever-smoke groups are shown in Table 1. The mean age of patients was 67.8 years. In addition, most of them were male 
Table 1

Comparison of demographic characteristics and underlying comorbidities between nonsmoker and smoker patients with bladder cancer

\begin{tabular}{|c|c|c|c|c|c|c|}
\hline & \multicolumn{3}{|c|}{ Before matching } & \multicolumn{3}{|c|}{ After matching } \\
\hline & $\begin{array}{l}\text { Never Smoking } \\
\quad(N=10634)\end{array}$ & $\begin{array}{l}\text { Ever Smoking } \\
\quad(N=5034)\end{array}$ & $P$ value & $\begin{array}{l}\text { Never Smoking } \\
\quad(N=4728)\end{array}$ & $\begin{array}{c}\text { Ever Smoking } \\
\quad(N=4728)\end{array}$ & $P$ value \\
\hline \multicolumn{7}{|l|}{ Variable, $n(\%)$} \\
\hline Age, Mean \pm SD & $70.53 \pm 12.26$ & $66.70 \pm 12.62$ & $<0.0001$ & $67.80 \pm 12.16$ & $67.72 \pm 12.22$ & 0.7478 \\
\hline \multicolumn{7}{|l|}{ Age group } \\
\hline$<50$ & $544(5.12)$ & $425(8.44)$ & $<0.0001$ & $340(7.19)$ & $329(6.96)$ & 0.8350 \\
\hline $50-59$ & $1372(12.90)$ & $1045(20.76)$ & & $810(17.13)$ & $849(17.96)$ & \\
\hline $60-69$ & $2741(25.78)$ & $1411(28.03)$ & & $1412(29.86)$ & $1398(29.57)$ & \\
\hline $70-79$ & $3246(30.52)$ & $1242(24.67)$ & & $1266(26.78)$ & $1242(26.27)$ & \\
\hline$>=80$ & $2731(25.68)$ & $911(18.10)$ & & $900(19.04)$ & $910(19.25)$ & \\
\hline \multicolumn{7}{|l|}{ Gender } \\
\hline Male & $6251(58.78)$ & 4889(97.12) & $<0.0001$ & 4584(96.95) & 4583(96.93) & 0.9524 \\
\hline Female & $4383(41.22)$ & $145(2.88)$ & & 144(3.05) & $145(3.07)$ & \\
\hline \multicolumn{7}{|l|}{ Clinical stage } \\
\hline $\begin{array}{l}\text { Non-muscle invasive } \\
\text { bladder cancer(NMIBC) }\end{array}$ & $7127(67.02)$ & $3378(67.10)$ & 0.9180 & $3323(70.28)$ & $3168(67.01)$ & 0.0006 \\
\hline $\begin{array}{l}\text { Muscle invasive bladder } \\
\text { cancer(MIBC) }\end{array}$ & $3507(32.98)$ & $1656(32.90)$ & & $1405(29.72)$ & $1560(32.99)$ & \\
\hline \multicolumn{7}{|l|}{$\mathrm{CCI}$} \\
\hline 0 & $4966(46.70)$ & $2776(55.15)$ & $<0.0001$ & $2485(52.56)$ & $2525(53.41)$ & 0.4595 \\
\hline $1-2$ & $3910(36.77)$ & $1647(32.72)$ & & $1608(34.01)$ & $1607(33.99)$ & \\
\hline$>=3$ & $1758(16.53)$ & $611(12.14)$ & & $635(13.43)$ & $596(12.61)$ & \\
\hline \multicolumn{7}{|l|}{ Comorbidity } \\
\hline Myocardial infarction & $128(1.20)$ & $89(1.77)$ & 0.0048 & $66(1.4)$ & $87(1.84)$ & 0.0870 \\
\hline Congestive heart failure & $510(4.80)$ & $183(3.64)$ & 0.0010 & $178(3.76)$ & $180(3.81)$ & 0.9142 \\
\hline Peripheral vascular disease & $150(1.41)$ & $74(1.47)$ & 0.7698 & $68(1.44)$ & $73(1.54)$ & 0.6714 \\
\hline Cardiovascular disease & $1033(9.71)$ & $468(9.30)$ & 0.4072 & $430(9.09)$ & $465(9.84)$ & 0.2189 \\
\hline Dementia & $485(4.56)$ & $133(2.64)$ & $<0.0001$ & $154(3.26)$ & $131(2.77)$ & 0.1665 \\
\hline $\begin{array}{l}\text { Chronic Obstructive } \\
\text { Pulmonary Disease }\end{array}$ & $914(8.60)$ & $614(12.20)$ & $<0.0001$ & $380(8.04)$ & $609(12.88)$ & $<0.0001$ \\
\hline Renal disease & $2229(20.96)$ & $570(11.32)$ & $<0.0001$ & $729(15.42)$ & $555(11.74)$ & $<0.0001$ \\
\hline Diabetes mellitus & $2793(26.26)$ & $1217(24.18)$ & 0.0051 & $1204(25.47)$ & $1181(24.98)$ & 0.5860 \\
\hline Hypertension & $3949(37.14)$ & $1663(22.04)$ & $<0.0001$ & $1690(35.74)$ & $1601(33.86)$ & 0.0547 \\
\hline Liver disease & $671(6.31)$ & $287(5.70)$ & 0.1375 & $316(6.68)$ & $268(5.67)$ & 0.0403 \\
\hline \multicolumn{7}{|l|}{ Treatment } \\
\hline Operation & 10182(95.75) & 4864(96.62) & 0.0089 & $4566(96.57)$ & $4565(96.55)$ & 0.9550 \\
\hline Radiotherapy & $985(9.26)$ & 449(8.92) & 0.4864 & 393(8.31) & 431(9.12) & 0.1659 \\
\hline Chemotherapy & $1354(12.73)$ & $750(14.90)$ & 0.0002 & $628(13.28)$ & $698(14.76)$ & 0.0382 \\
\hline $\mathrm{RT}$ or $\mathrm{CT}$ & $1864(17.53)$ & $951(18.89)$ & 0.0380 & $803(16.98)$ & $896(18.95)$ & 0.0127 \\
\hline Drinking & $82(0.77)$ & $708(14.06)$ & $<0.0001$ & $55(1.16)$ & $683(14.45)$ & $<0.0001$ \\
\hline Chewing betel nut & $66(0.62)$ & $895(17.78)$ & $<0.0001$ & $44(0.93)$ & $828(17.51)$ & $<0.0001$ \\
\hline \multicolumn{7}{|l|}{ BMI } \\
\hline$<18.5$ & $955(8.98)$ & $338(6.71)$ & $<0.0001$ & $295(6.24)$ & $329(6.96)$ & 0.3104 \\
\hline 18.5 to 25 & $5625(52.90)$ & $2618(52.01)$ & & $2447(51.76)$ & $2456(51.95)$ & \\
\hline$\geq 25$ & $4054(38.12)$ & $2078(41.28)$ & & $1986(42.01)$ & 1943(41.10) & \\
\hline Time to follow up, Mean \pm SD & $2.42 \pm 1.89$ & $2.60 \pm 1.89$ & $<0.0001$ & $2.65 \pm 1.92$ & $2.55 \pm 1.88$ & 0.0157 \\
\hline Overall mortality & $2945(27.69)$ & $1340(26.62)$ & 0.1586 & $1137(24.05)$ & $1294(27.37)$ & 0.0002 \\
\hline Time to death, Mean \pm SD & $1.47 \pm 1.35$ & $1.64 \pm 1.41$ & 0.0004 & $1.65 \pm 1.40$ & $1.64 \pm 1.42$ & 0.7919 \\
\hline Cancer specific mortality & $1572(14.78)$ & $684(13.59)$ & 0.0466 & $579(12.25)$ & $657(13.9)$ & 0.0173 \\
\hline Time to death, Mean \pm SD & $1.23 \pm 1.12$ & $1.41 \pm 1.22$ & 0.0013 & $1.39 \pm 1.17$ & $1.41 \pm 1.23$ & 0.7115 \\
\hline
\end{tabular}

(96.9\%). Moreover, patients who were ever smokers showed a higher incidence of drinking wine and consuming betel nut than those who were never smokers $(14.45 \%$ and $17.51 \%$, respectively; $P<0.0001)$. More patients who were ever smokers were at the last clinical cancer stages $(2,3,4$; MIBC) compared to those who were never smokers (32.99\% vs. $29.72 \%$;
$P=0.0006)$. Overall and specific mortality rates were significantly higher in patients who were ever smokers than in those who were never smokers (overall, $27.37 \%$ vs. $24.05 \%$; $P=0.0002$; specific, $13.9 \%$ vs. $12.25 \% ; P=0.0173$ ).

Table 2 displays the finding that smoking is an independent risk factor for the overall and specific 


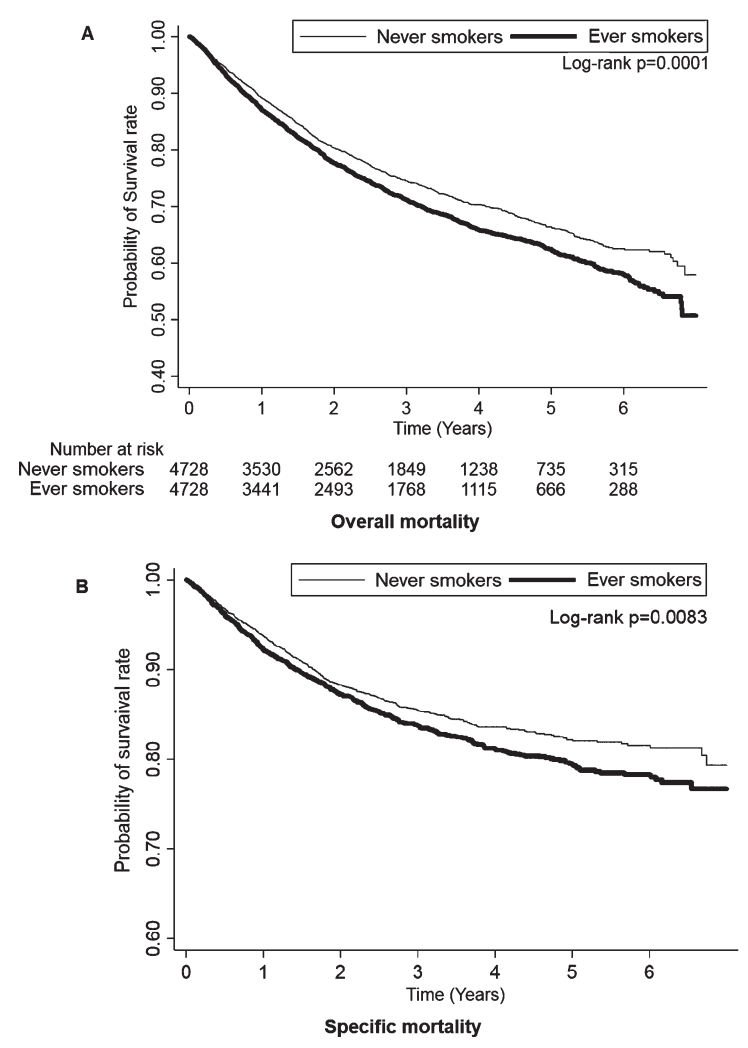

Fig. 2. Kaplan-Meier plot of overall mortality among never smokers and ever smokers.

mortalities in patients with bladder cancer. The overall mortality rate in the patients who were ever smokers was 1.15 -fold (95\% confidence interval [CI], 1.06-1.26, $P=0.0014$ ) adjusted HR compared with that of those who were never smokers. In addition, the specific mortality rate of the patients who were ever smokers was 1.16-fold (95\% CI, 1.03-1.30, $P=0.0176)$ adjusted HR compared with those who were never smokers. The result was compatible with the Kaplan-Meier plotted the trend of overall and specific mortality between never smokers and ever smokers, as shown in Fig. 2. The overall and specific mortality rates were significantly higher in the patients who were ever smokers than in those who were never smokers (log-rank test, $P=0.0001$ and $P=0.0083$, respectively) during the follow-up period.

Table 2 also summarizes the risk factors for the overall and specific mortality rates of patients with bladder cancer. There was a significantly higher overall mortality rate in patients with bladder cancer with an underweight BMI $\{<18.5$; adjusted HR (95\% CI): 1.65 (1.46-1.87); $P<0.0001\}$, a higher CCI $\{1-2$ or $\geq 3$; adjusted HR (95\% CI): 1.14
(1.01-1.29), 1.28 (1.04-1.59); $P=0.0315$ and 0.023$\}$, and MIBC \{adjusted HR (95\% CI): 3.40 (3.09-3.73); $P<0.0001\}$. In addition, there was a significantly higher cancer-specific mortality rate in bladder cancer patients with an underweight BMI $\{<18.5$; adjusted HR (95\% CI): 1.54 (1.29-1.84); $P<0.0001\}$, and MIBC \{adjusted HR (95\% CI): 6.71 (5.82-7.74); $P<0.0001\}$.

Table 3 shows the risk factors for the overall and specific mortalities associated with ever-smoke bladder cancer patients. Compared with never-smoke male patients, ever-smoke male patients exhibited a 1.16-fold and 1.17-fold adjusted HRs of overall and specific mortality rates $(P=0.0007$ and 0.0145 , respectively). Ever-smoke bladder cancer patients also had an increased overall and specific mortality rates by 1.31 -fold and 1.55 -fold adjusted HRs compared with non-smoking bladder cancer patients among those with a $\mathrm{CCI} \geq 3(P=0.0119$ and 0.0092 , respectively). Moreover, ever-smoke NMIBC patients exhibited a 1.21-fold and a 1.47-fold adjusted HRs for the overall and specific mortality rates compared with those of never-smoke patients $(P=0.0038$ and 0.0014 , respectively). Furthermore, we followed up comorbidity risks for bladder cancer; results are listed in Appendix 2. Having DM resulted in a 1.27-fold and a 1.28-fold adjusted HRs in the overall and specific mortality of ever-smoke patients $(P=0.0046$ and 0.0419 , respectively). Having hypertension also resulted in a 1.19-fold adjusted HRs in the overall mortality of ever-smoke patients $(P=0.0105)$. Regardless of the subsequent treatment for patients with bladder cancer, smoking increased the risk for overall mortality, and significant differences exist in operation and chemotherapy. (1.10-fold and 1.25 -fold in the overall mortality, respectively; $P=0.0387$ and 0.0163 ).

\section{DISCUSSION}

Bladder cancer is a common cancer worldwide. Previous studies reported that cigarette smoking may lead to a large primary tumor size, a higher clinical stage, and a higher pathological grade upon initial diagnosis of bladder cancer [18, 19]. In addition, smoking also contributes to the development of bladder cancer [3, 4] and influences outcomes. Over the past few years, several reviews investigated the course of smoking-influenced bladder cancer [7-10]. However, most studies investigated the oncological outcomes in patients receiving TURBT 
Table 2

Overall risk factor analysis of overall or specific mortality for patients with bladder cancer

\begin{tabular}{|c|c|c|c|c|c|c|c|c|}
\hline & \multicolumn{4}{|c|}{ Overall mortality } & \multicolumn{4}{|c|}{ Specific mortality } \\
\hline & $\begin{array}{l}\text { Crude HR } \\
(95 \% \mathrm{CI})\end{array}$ & $p$-value & $\begin{array}{c}\text { Adjusted HR } \\
(95 \% \mathrm{CI})\end{array}$ & $p$-value & $\begin{array}{l}\text { Crude HR } \\
(95 \% \mathrm{CI})\end{array}$ & $p$-value & $\begin{array}{c}\text { Adjusted HR } \\
(95 \% \mathrm{CI})\end{array}$ & $p$-value \\
\hline \multicolumn{9}{|l|}{ Smoking } \\
\hline Never & Ref. & & Ref. & & Ref. & & Ref. & \\
\hline Ever/ Current & $1.17(1.08-1.27)$ & $<0.0001$ & $1.15(1.06-1.26)$ & 0.0014 & $1.16(1.04-1.30)$ & 0.0085 & $1.16(1.03-1.30)$ & 0.0176 \\
\hline \multicolumn{9}{|l|}{ Drinking } \\
\hline Never & Ref. & & Ref. & & Ref. & & Ref. & \\
\hline Ever/ Current & $1.42(1.24-1.62)$ & $<0.0001$ & $1.05(0.91-1.22)$ & 0.4963 & $1.15(0.94-1.41)$ & 0.1725 & $0.84(0.67-1.05)$ & 0.1190 \\
\hline \multicolumn{9}{|l|}{ Chewing betel nut } \\
\hline Never & Ref. & & Ref. & & Ref. & & Ref. & \\
\hline Ever/ Current & $1.09(0.95-1.25)$ & 0.2159 & $1.08(0.93-1.25)$ & 0.3253 & $1.08(0.90-1.31)$ & 0.4091 & $1.04(0.85-1.29)$ & 0.6855 \\
\hline \multicolumn{9}{|l|}{ BMI } \\
\hline$<18.5$ & $2.15(1.90-2.42)$ & $<0.0001$ & $1.65(1.46-1.87)$ & $<0.0001$ & $2.08(1.75-2.48)$ & $<0.0001$ & $1.54(1.29-1.84)$ & $<0.0001$ \\
\hline $18.5-25$ & Ref. & & Ref. & & Ref. & & Ref. & \\
\hline$\geq 25$ & $0.60(0.55-0.66)$ & $<0.0001$ & $0.70(0.64-0.77)$ & $<0.0001$ & $0.63(0.56-0.72)$ & $<0.0001$ & $0.75(0.66-0.75)$ & $<0.0001$ \\
\hline \multicolumn{9}{|c|}{$0.00(0.03-0.00)$} \\
\hline 0 & Ref. & & Ref. & & Ref. & & Ref. & \\
\hline $1-2$ & $1.63(1.50-1.79)$ & $<0.0001$ & $1.14(1.01-1.29)$ & 0.0315 & $1.28(1.13-1.45)$ & $<0.0001$ & $1.04(0.88-1.24)$ & 0.6406 \\
\hline$>=3$ & $2.68(2.40-2.99)$ & $<0.0001$ & $1.28(1.04-1.59)$ & 0.0230 & $1.69(1.43-1.99)$ & $<0.0001$ & $1.03(0.75-1.42)$ & 0.8596 \\
\hline \multicolumn{9}{|l|}{ Comorbidity } \\
\hline MI & $1.30(0.98-1.73)$ & 0.0713 & $0.97(0.72-1.31)$ & 0.8582 & $1.00(0.64-1.57)$ & 1.0000 & $0.88(0.55-1.41)$ & 0.6021 \\
\hline $\mathrm{CHF}$ & $2.39(2.03-2.80)$ & $<0.0001$ & $1.65(1.39-1.97)$ & $<0.0001$ & $1.81(1.41-2.33)$ & $<0.0001$ & $1.59(1.21-2.08)$ & 0.0009 \\
\hline PVD & $1.72(1.30-2.27)$ & 0.0001 & $1.26(0.94-1.67)$ & 0.1172 & $1.35(0.88-2.08)$ & 0.1689 & $1.19(0.77-1.86)$ & 0.4337 \\
\hline CVD & $1.71(1.53-1.92)$ & $<0.0001$ & $1.17(1.02-1.34)$ & 0.0213 & $1.31(1.09-1.56)$ & 0.0031 & $1.06(0.86-1.31)$ & 0.5621 \\
\hline Dementia & $2.62(2.21-3.09)$ & $<0.0001$ & $1.53(1.28-1.84)$ & $<0.0001$ & $2.48(1.95-3.14)$ & $<0.0001$ & $1.86(1.43-2.41)$ & $<0.0001$ \\
\hline COPD & $1.81(1.62-2.02)$ & $<0.0001$ & $1.09(0.95-1.24)$ & 0.2213 & $1.43(1.21-1.69)$ & $<0.0001$ & $1.03(0.84-1.25)$ & 0.8092 \\
\hline Renal disease & $1.82(1.65-2.02)$ & $<0.0001$ & $1.50(1.29-1.74)$ & $<0.0001$ & $1.41(1.21-1.64)$ & $<0.0001$ & $1.40(1.12-1.74)$ & 0.0035 \\
\hline $\mathrm{DM}$ & $1.28(1.17-1.40)$ & $<0.0001$ & $1.15(1.05-1.27)$ & 0.0044 & $1.19(1.05-1.35)$ & 0.0057 & $1.18(1.03-1.35)$ & 0.0209 \\
\hline Hypertension & $1.25(1.15-1.25)$ & $<0.0001$ & $1.00(0.91-1.08)$ & 0.9133 & $1.10(0.98-1.23)$ & 0.1121 & $0.95(0.84-1.08)$ & 0.4452 \\
\hline Liver disease & $1.19(1.02-1.40)$ & 0.0318 & $1.25(1.05-1.49)$ & 0.0122 & $0.76(0.57-1.00)$ & 0.0462 & $0.91(0.68-1.23)$ & 0.5320 \\
\hline \multicolumn{9}{|l|}{ Treatment } \\
\hline $\mathrm{OP}$ & $0.25(0.22-0.29)$ & $<0.0001$ & $0.45(0.38-0.52)$ & $<0.0001$ & $0.22(0.18-0.26)$ & $<0.0001$ & $0.46(0.38-0.56)$ & $<0.0001$ \\
\hline RT & $3.04(2.74-3.37)$ & $<0.0001$ & $1.06(0.94-1.19)$ & 0.3711 & $4.62(4.07-5.26)$ & $<0.0001$ & $1.23(1.07-1.42)$ & 0.0039 \\
\hline $\mathrm{CT}$ & $2.31(2.10-2.54)$ & $<0.0001$ & $1.22(1.10-1.36)$ & 0.0002 & $3.78(3.36-4.25)$ & $<0.0001$ & $1.42(1.24-1.62)$ & $<0.0001$ \\
\hline \multicolumn{9}{|l|}{ Clinical stage } \\
\hline NMIBC & Ref. & & Ref. & & Ref. & & Ref. & \\
\hline MIBC & $3.91(3.61-4.24)$ & $<0.0001$ & $3.40(3.09-3.73)$ & $<0.0001$ & $8.64(7.61-9.83)$ & $<0.0001$ & $6.71(5.82-7.74)$ & $<0.0001$ \\
\hline
\end{tabular}

*MI: Myocardial infarction; CHF: Congestive heart failure; PVD: Peripheral vascular disease; CVD: Cardiovascular disease; COPD: Chronic Obstructive Pulmonary Disease; DM: Diabetes mellitus, OP: Operation;RT: Radiotherapy;CT: Chemotherapy; NMIBC: non-muscle invasive bladder cancer; MIBC: muscle invasive bladder cancer. ** Adjusted for clinical stage, smoking, drinking, chewing betel nut, BMI, CCI, comorbidity(include MI, CHF, PVD, CVD, Dementia, COPD, Renal disease, DM, Hypertension and liver disease) and treatment(include OP, RT and CT). 
Table 3

Comparison of the risk for overall or specific mortality between nonsmoker and smoker patients with bladder cancer stratified by gender, age group, $\mathrm{CCI}$ and clinical stage

\begin{tabular}{|c|c|c|c|c|c|c|c|}
\hline & \multirow[b]{2}{*}{ Smoker } & \multirow[b]{2}{*}{ Death } & \multicolumn{2}{|c|}{ Overall mortality } & \multirow[b]{2}{*}{ Death in $\mathrm{BC}$} & \multicolumn{2}{|c|}{ Specific mortality } \\
\hline & & & $\begin{array}{c}\text { Adjusted HR } \\
(95 \% \mathrm{CI})\end{array}$ & $p$-value & & $\begin{array}{l}\text { Adjusted HR } \\
(95 \% \mathrm{CI})\end{array}$ & $p$-value \\
\hline Overall & 4728 & 1294 & $1.15(1.06-1.26)$ & 0.0014 & 657 & $1.16(1.03-1.30)$ & 0.0176 \\
\hline \multicolumn{8}{|l|}{ Stratified } \\
\hline \multicolumn{8}{|l|}{ Gender } \\
\hline Male & 4583 & 1255 & $1.16(1.07-1.27)$ & 0.0007 & 636 & $1.17(1.03-1.32)$ & 0.0145 \\
\hline Female & 145 & 39 & $0.90(0.50-1.61)$ & 0.7113 & 21 & $0.97(0.45-2.09)$ & 0.9323 \\
\hline \multicolumn{8}{|l|}{ Age group } \\
\hline$<50$ & 329 & 28 & $0.80(0.45-1.42)$ & 0.4458 & 20 & $0.81(0.42-1.54)$ & 0.5175 \\
\hline $50-59$ & 849 & 116 & $1.10(0.82-1.47)$ & 0.5358 & 77 & $1.06(0.74-1.51)$ & 0.7718 \\
\hline $60-69$ & 1398 & 274 & $1.20(0.99-1.45)$ & 0.0619 & 151 & $1.27(0.98-1.65)$ & 0.0681 \\
\hline $70-79$ & 1242 & 407 & $1.20(1.03-1.40)$ & 0.0223 & 207 & $1.24(1.00-1.55)$ & 0.0548 \\
\hline$>=80$ & 910 & 469 & $1.13(0.98-1.30)$ & 0.0196 & 202 & $1.05(0.85-1.30)$ & 0.6798 \\
\hline \multicolumn{8}{|l|}{ CCI } \\
\hline 0 & 2525 & 559 & $1.13(1.00-1.29)$ & 0.0588 & 330 & $1.13(0.96-1.34)$ & 0.1546 \\
\hline $1-2$ & 1607 & 490 & $1.08(0.94-1.24)$ & 0.2837 & 229 & $1.04(0.85-1.27)$ & 0.7157 \\
\hline$>=3$ & 596 & 245 & $1.31(1.06-1.61)$ & 0.0119 & 98 & $1.55(1.12-2.15)$ & 0.0092 \\
\hline \multicolumn{8}{|c|}{ Clinical stage } \\
\hline NMIBC & 3168 & 566 & $1.21(1.06-1.38)$ & 0.0038 & 177 & $1.47(1.16-1.86)$ & 0.0014 \\
\hline MIBC & 1560 & 728 & $1.11(0.99-1.25)$ & 0.0694 & 480 & $1.07(0.93-1.23)$ & 0.3544 \\
\hline
\end{tabular}

*MI: Myocardial infarction; CHF: Congestive heart failure; PVD: Peripheral vascular disease; CVD: Cardiovascular disease; COPD: Chronic Obstructive Pulmonary Disease; DM: Diabetes mellitus, OP: Operation;RT: Radiotherapy;CT: Chemotherapy; NMIBC: non-muscle invasive bladder cancer; MIBC: muscle invasive bladder cancer. ${ }^{* *}$ Adjusted for clinical stage, smoking, drinking, chewing betel nut, BMI, CCI, comorbidity (include MI, CHF, PVD, CVD, Dementia, COPD, Renal disease, DM, Hypertension and liver disease) and treatment(include operation, radiotherapy and chemotherapy).

operations NMIBC or RC in MIBC. They only reported that cigarette smoking exposure increased the risk of disease recurrence. Conclusive evidence to support the relationship of smoking and the overall or cancer-specific mortality remains lacking. In the present study, the ever-smoke patients had higher clinical stages at initial diagnosis of bladder cancer $(P=0.0006)$ and had significantly worse overall and specific mortality rates $(P=0.002$ and 0.017 , respectively) compared with never-smoke patients. Subgroup analyses of NMIBC and MIBC revealed that the patients who were ever smokers had significantly increased overall and specific mortality rates $(P=0.0039$ and 0.0015 , respectively) in NMIBC. Although patients with MIBC who were ever smokers had a similar outcome that increased by 1.12 -fold in terms of overall mortality and by 1.07 -fold in terms of cancer-specific mortality, the difference was not significant $(P=0.0553$ and 0.3669 , respectively). As shown in Appendix 3, the patients who were ever smokers receiving TURBT in NMIBC had worse overall and cancer-specific mortality rates compared with patients who were never smokers $(P=0.0069$ and 0.0026, respectively). Cigarette smoking exerted no significant influence on any treatment in MIBC. In general, bladder cancer is a complex and heterogeneous disease. Subset analyses of particular clinical stages with different treatment modalities are currently lacking. Therefore, several study-related and general limitations should be addressed in future research.

Urologists must identify risk factors that can predict cancer progression, including cancer-specific and all-cause mortality rates, to help in patient counseling and to select further treatment $[14,15]$. The present study identified risk factors for overall and cancer-specific mortalities associated with bladder cancer patients who were ever-smokers. They had a significantly higher overall and cancerspecific mortality rates, especially men, those with a CCI $\geq 3$, those with a DM comorbidity, and those with NMIBC. Factors for sex differences in bladder cancer incidence and survival, which was higher in men, were related to a few factors likely acting in combination, such as differences in access to health care, delayed diagnosis probably due to hematuria, or lower urinary tract symptoms being attributed to cystitis in women, occupational exposure, and smoking patterns $[16,17]$. We identified several studies of sex-specific differential effects of smoking in patients with bladder cancer treated with TURBT or RC. In a cohort of 286 patients, Fleshner et al.[20] determined that continued smoking in men was associated with a diminished time to recurrence (HR: 1.40; 
95\% CI, 1.03-1.91) and diminished survival free of adverse events $(P=0.14)$. Rink et al. [8] reported that female smokers receiving $\mathrm{RC}$ for bladder cancer were more likely to experience disease recurrence (HR: $1.58, P<0.001$ ), cancer-specific mortality (HR: $1.67, P<0.001$ ), and any cause mortality (HR: 1.63 , $P<0.001)$ compared with their male counterparts. These findings suggested that the effects of smoking on patient outcomes occurred more in one sex compared with the other [21]. In the present study, for the higher smoking prevalence rate of men in Taiwan (males: $23.4 \%$ and females: $2.4 \%$ ) [2], we adjusted for confounding factors such as age and sex by using the multivariate analysis step-bystep to reduce these biases. As shown in Table 2, women with bladder cancer had higher overall and specific mortality rates than men, but there was no significant difference. However, ever-smoke men with bladder cancer showed significantly higher overall and specific mortality rates than their never-smoke counterparts, as shown in Table 3 ( $P=0.0007$ and 0.0145 , respectively).

The CCI is a tool used for calculating comorbidities and therefore mortality risk. For bladder cancer, a higher CCI was associated with a higher mortality rate [22]. Ever-smoke patients had a significantly higher mortality rate. DM was also a risk factor for bladder cancer. It was associated with cancer incidence and poor prognosis [23-25]. In the present study, bladder cancer patients with DM who were ever-smokers had a multiplier effect, which significantly increased the overall and specific mortality rates.

Several large cohorts and case-control studies noted that smoking cessation enabled an immediate decrease in risk for bladder cancer [26]. Smoking cessation and the time since quitting favorably affected the risk for bladder cancer incidence and the prognosis $[8,27]$. For many patients, a cancer diagnosis motivated them to successfully quit smoking. Patients with bladder cancer were often willing to quit smoking with the help of their physicians [28]. A survey of 601 urologists found that more than half of them never discussed smoking cessation with their patients, and almost one in four believed that cessation did not impact disease prognosis [29]. Appropriate patient education and physician meetings may increase the probability to quit smoking, improve outcomes, and enhance the quality of life [30]. Urologists must focus on smoking cessation and help patients stop smoking as soon as possible.

This study has several limitations. The retrospective nationwide database did not contain detailed smoking information, such as smoking duration, age of first exposure, number of cigarettes smoked, date of cessation, tobacco type, and whether smoking cessation occurred after diagnosis [27-32]. Tumorrelated information was also lacking, including tumor histological data, different treatments in each stage, and recurrence condition. Further, some risk factors that are often associated with bladder cancer were unable to be included, such as environmental exposure, family history, and socioeconomic status $[33,34]$. Due to the small proportion of women with bladder cancer [1] and the low population of female smoking in Taiwan [2], there were few female patients after matching in our study. Regarding women, the correlation between smoking and mortality will need to be investigated further in the future. Last, all patients were treated in the Taiwanese medical system, indicating a potential lack of generalizability, although some studies have confirmed the accuracy of the information in Taiwan's National Health Insurance Research Database [35], and some topics which were discussed in our study have been published internationally, such as bladder cancer [36] and smoking [37].

In conclusion, smoking behavior directly contributes to the increased overall and cancer-specific mortality rates of patients with bladder cancer. It is also a risk factor along with other factors such as a male sex, a CCI $\geq 3$, a DM comorbidity, and NMIBC.

\section{ACKNOWLEDGMENTS}

We are grateful to Health Data Science Center, National Cheng Kung University Hospital for providing administrative and technical support. The author wishes to acknowledge to Dr. CC Tseng, YH, Shen, YP, Huang for guidance.

\section{FUNDING}

This study was supported by the grants from Chi Mei Medical Center CMFHR11060.

\section{AUTHOR CONTRIBUTIONS}

All authors have made substantial contributions to the work, have approved the final version to be published and have agreed to be accountable for the accuracy and integrity of the work. Chung-Han Ho - conception; performance of work; interpretation and analysis of data; writing the article. Wen-Hsin 
Tseng - conception; performance of work; interpretation and analysis of data; writing the article. Steven K. Huang - interpretation and analysis of data; writing the article. Chien-Liang Liu - interpretation and analysis of data; writing the article. Yu-Cih Wu - interpretation and analysis of data; writing the article. Allen W.Chiu - interpretation and analysis of data; writing the article. Khaa Hoo Ong- conception; performance of work; interpretation and analysis of data; writing the article.

\section{CONFLICT OF INTEREST}

$\mathrm{CH}, \mathrm{WT}, \mathrm{SH}, \mathrm{CL}, \mathrm{YW}, \mathrm{AC}$ and $\mathrm{KO}$ have no conflicts of interest to declare.

\section{REFERENCES}

[1] Siegel RL, Miller KD, Fuchs HE, et al. Cancer Statistics, 2021. CA Cancer J Clin 2021;71:7-33.

[2] Ministry of Health and Welfare, ROC (Taiwan), 2019 Taiwan Health and Welfare Report [cited 2021 May 02]. Available from: https://www.mohw.gov.tw/fp-137-528782.html

[3] Rink M, Crivelli JJ, Shariat SF, et al. Smoking and Bladder Cancer: A Systematic Review of Risk and Outcomes. Eur Urol Focus 2015;1:17-27.

[4] Jiang X, Castelao JE, Yuan JM, et al. Cigarette smoking and subtypes of bladder cancer. Int J Cancer 2012;130:896-901.

[5] Jha P, Peto R. Global effects of smoking, of quitting, and of taxing tobacco. N Engl J Med 2014;370:60-68.

[6] National Comprehensive Cancer Network. Bladder Cancer (Version 3.2021) [cited 2021 May 02]. Available from: https://www.nccn.org/guidelines/recently-publishedguidelines

[7] Rink M, Furberg H, Zabor EC, et al. Impact of smoking and smoking cessation on oncologic outcomes in primary nonmuscle-invasive bladder cancer. Eur Urol 2013;63:724-32.

[8] Rink M, Zabor EC, Furberg H, et al. Impact of smoking and smoking cessation on outcomes in bladder cancer patients treated with radical cystectomy. Eur Urol 2013;64:456-64.

[9] Cacciamani GE, Ghodoussipour S, Mari A, et al. Association between Smoking Exposure, Neoadjuvant Chemotherapy Response and Survival Outcomes following Radical Cystectomy: Systematic Review and Meta-Analysis. J Urol 2020;204:649-60.

[10] Rink M, Xylinas E, Babjuk M, et al. Impact of smoking on outcomes of patients with a history of recurrent nonmuscle invasive bladder cancer. J Urol 2012;188:2120-7.

[11] Austin PC. An Introduction to Propensity Score Methods for Reducing the Effects of Confounding in Observational Studies. Multivariate Behav Res 2011;46:399-424.

[12] Austin PC. Balance diagnostics for comparing the distribution of baseline covariates between treatment groups in propensity-score matched samples. Stat Med 2009;28:3083107.

[13] Parsons LS, Ovation Research Group: Performing a 1:N CaseControl Match on Propensity Score. Proceedings of the 29th SAS Users Group International, Montreal, Canada, 2004, pp 1-11
[14] Williams SB, Kamat AM, Chamie K, et al. Systematic Review of Comorbidity and Competing-risks Assessments for Bladder Cancer Patients. Eur Urol Oncol 2018;1:91-100.

[15] Zhang L, Wu B, Zha Z, et al. Clinicopathological factors in bladder cancer for cancer-specific survival outcomes following radical cystectomy: a systematic review and metaanalysis. BMC Cancer 2019;19:716.

[16] Burger M, Catto JW, Dalbagni G, et al. Epidemiology and risk factors of urothelial bladder cancer. Eur Urol 2013;63:234-41.

[17] Cumberbatch MGK, Jubber I, Black PC, et al. Epidemiology of Bladder Cancer: A Systematic Review and Contemporary Update of Risk Factors in 2018. Eur Urol 2018;74: 784-95.

[18] van Roekel EH, Cheng KK, James ND, et al. Smoking is associated with lower age, higher grade, higher stage, and larger size of malignant bladder tumors at diagnosis. Int $\mathrm{J}$ Cancer 2013;133:446-54.

[19] Chu W-C, Chen C-H. Smoking behavior and survival outcomes in bladder cancer patients. Urological Science 2020;31:123.

[20] Fleshner N, Garland J, Moadel A, et al. Influence of smoking status on the disease-related outcomes of patients with tobacco-associated superficial transitional cell carcinoma of the bladder. Cancer 1999;86:2337-45.

[21] Yadav PK, Yang E, Fan N, et al. Impact of Smoking on Prognosis and Survival of Patients with Bladder Cancer. Archives of Nephrology and Urology 2019;2:80-92.

[22] Goossens-Laan CA, Leliveld AM, Verhoeven RH, et al. Effects of age and comorbidity on treatment and survival of patients with muscle-invasive bladder cancer. Int J Cancer 2014;135:905-12.

[23] Zhu Z, Wang X, Shen Z, et al. Risk of bladder cancer in patients with diabetes mellitus: an updated meta-analysis of 36 observational studies. BMC Cancer 2013;13:310.

[24] Xu Y, Huo R, Chen X, et al. Diabetes mellitus and the risk of bladder cancer: A PRISMA-compliant meta-analysis of cohort studies. Medicine (Baltimore) 2017;96:e8588.

[25] Pan Y, Lee CY, Lee LM, et al. Incidence of Bladder Cancer in Type 2 Diabetes Mellitus Patients: A Population-Based Cohort Study. Medicina (Kaunas) 2020;56.

[26] Soria F, Marra G, Čapoun O, et al. Prevention of bladder cancer incidence and recurrence: tobacco use. Curr Opin Urol 2018;28:80-7.

[27] Mori K, Mostafaei H, Abufaraj M, et al. Smoking and bladder cancer: review of the recent literature. Curr Opin Urol 2020;30:720-5.

[28] Bassett JC, Gore JL, Chi AC, et al. Impact of a bladder cancer diagnosis on smoking behavior. J Clin Oncol 2012;30:1871-8

[29] Bjurlin MA, Goble SM, Hollowell CM. Smoking cessation assistance for patients with bladder cancer: a national survey of American urologists. J Urol 2010;184:1901-6.

[30] Kowalkowski MA, Goltz HH, Petersen NJ, et al. Educational opportunities in bladder cancer: increasing cystoscopic adherence and the availability of smokingcessation programs. J Cancer Educ 2014;29:739-45.

[31] McCormack VA, Agudo A, Dahm CC, et al. Cigar and pipe smoking and cancer risk in the European Prospective Investigation into Cancer and Nutrition (EPIC). Int J Cancer 2010;127:2402-11.

[32] van Osch FH, Jochems SH, van Schooten FJ, et al. Quantified relations between exposure to tobacco smoking and bladder cancer risk: a meta-analysis of 89 observational studies. Int J Epidemiol 2016;45:857-70. 
[33] Zaitsu M, Lee HE, Lee S, et al. Occupational disparities in bladder cancer survival: A population-based cancer registry study in Japan. Cancer Med 2020;9:894-901.

[34] Lee HE, Zaitsu M, Kim EA, et al. Occupational Class and Cancer Survival in Korean Men: Follow-Up Study of Nation-Wide Working Population. Int J Environ Res Public Health 2020;17:303.

[35] Hsieh CY, Su CC, Shao SC, et al. Taiwan's National Health Insurance Research Database: past and future. Clin Epidemiol 2019;11:349-58.
[36] Pan Y, Lee CY, Lee LM, et al. Incidence of Bladder Cancer in Type 2 Diabetes Mellitus Patients: A Population-Based Cohort Study. Medicina (Kaunas) 2020;56:441.

[37] Chung WS, Kung PT, Chang HY, et al. Demographics and medical disorders associated with smoking: a populationbased study. BMC Public Health 2020;20:702. 